\title{
ESTUDO DE MERCADO DE BISCOITO TIPO COOKIE INTEGRAL ADICIONADO DE GOMA DE LINHAÇA DOURADA
}

\author{
Isabella Varanda Oliveira ${ }^{1}$ \\ Andressa Ladeira Bernardes ${ }^{2}$ \\ Wallaf Costa Vimercati ${ }^{3}$ \\ Suzana Maria Della Lucia ${ }^{4}$ \\ André Gustavo Vasconcelos Costa ${ }^{5}$
}

\begin{abstract}
Resumo: O interesse por produtos mais saudáveis está se tornando cada vez maior entre os consumidores. Este fato tem levado pesquisadores e a indústria de alimentos a procurarem maneiras de desenvolverem alimentos que atendam às exigências dos consumidores, como por exemplo, produtos com acrescidos de nutrientes que proporcionem benefícios à saúde e ao estado nutricional. Realizou-se uma pesquisa de mercado com 68 alunos e funcionários do Centro de Ciências Agrárias e Engenharias da Universidade Federal do Espírito Santo, para verificar a aceitabilidade de um cookie integral com goma de linhaça. Este, por sua vez, seria um produto rico em fibras, ômega 3 e reduzido em gordura, visto que a goma de linhaça seria usada em substituição ao ovo. Observou-se que o consumo de linhaça e produtos integrais é feito por grande parte dos entrevistados, 56\% e 78\% respectivamente. Em relação à aquisição, $69 \%$ dos entrevistados talvez compraria, e $31 \%$ definitivamente compraria este produto.
\end{abstract}

Palavras-chave: Desenvolvimento de novos produtos; Cookie; Gel de linhaça; Produto integral.

\footnotetext{
${ }^{1}$ Programa de Pós-graduação em Ciência e Tecnologia de Alimentos/Universidade Federal do Espírito Santo, Brasil. E-mail: isabella.varanda@gmail.com.

2 Programa de Pós-graduação em Ciência e Tecnologia de Alimentos/Universidade Federal do Espírito Santo, Brasil. E-mail: andressa.bernardes@hotmail.com.

3 Programa de Pós-graduação em Ciência e Tecnologia de Alimentos/Universidade Federal do Espírito Santo, Brasil. E-mail: wallafcosta@hotmail.com.

4 Professor no Programa de Pós-graduação em Ciência e Tecnologia de Alimentos/Universidade Federal do Espírito Santo, Brasil. E-mail: smdlucia@yahoo.com.br.

5 Professor no Programa de Pós-graduação em Ciência e Tecnologia de Alimentos/Universidade Federal do Espírito Santo, Brasil. E-mail: agvcosta@yahoo.com.br.
} 